\title{
04 EFFECTIVENESS OF WEB-BASED EXPERT SYSTEM FOR SUBSTANCE ABUSE PREVENTION IN KOREAN
} ADOLESCENTS

doi:10.1136/injuryprev-2012-0405900.4

${ }^{1} \mathrm{JY}$ Bae, ${ }^{2} \mathrm{AC}$ Bae. ${ }^{1}$ International Safe Community Research Centre of Busan

Metropolitan and Professor, Inje University, Korea; ${ }^{2}$ Seoul National University, Korea

Background Substance abuse in adolescents is one of the most serious health problems in Korea. Recently, adolescent's smoking, drinking and drug abuse increasing rapidly.

Purposes There were two purposes of this study. First was to describe the development process of a user-centered, web-based expert system for substance abuse prevention with the following objectives: (i) to empower adolescent by informing them how to prevent and manage substance abuse, (ii) to provide evidence-based interventions. Another purpose of this study was to examine the 
effectiveness of this system on smoking and drinking behaviour in adolescents.

Methods Development process included four distinct phases: a needs assessment, analysis, design/development/testing, and the application release. After development of the system, the effectiveness was examined with forty adolescents who were recruited from the community. The non-equivalent control group pretest-posttest design was used.

Results The web site was released using the URL: http://www. baejy.com/yebang/. This system includes intervention programmes to relieve substance abuse such as preventive education, cognitive behavioural therapy, assertiveness training, anger management, self confidence training and family therapy. After the 8-week period of intervention, experimental group showed a significant decrease in their smoking and drinking behaviour.

Contribution to the Field The result was a web-based expert system for substance abuse prevention with a high degree of usability with an evidence-based intervention. The author believes that this development design will be utilised to create safety education programmes suitable for other safety education programme as well as high-risk populations. 